\title{
Compressed Sensing-based Multiple-target Tracking Algorithm for Ad Hoc Camera Sensor Networks
}

\author{
$\mathrm{Xu} \mathrm{Lu}{ }^{1 *}$, Lianglun Cheng ${ }^{2}$, Jun Liu ${ }^{1}$, Rongjun Chen ${ }^{3}$ \\ ${ }^{1}$ School of Automatization, Guangdong Polytechnic Normal University \\ Guangzhou 510665, China \\ [bruda@126.com] \\ ${ }^{2}$ School of Automatization, Guangdong University of Technology \\ Guangzhou 510006, China \\ [llcheng@gdut.edu.cn] \\ ${ }^{3}$ School of Computer Science, Guangdong Polytechnic Normal University \\ Guangzhou 510665, China \\ [46812659@qq.com] \\ *Corresponding author: Xu Lu \\ Received April 21, 2017; revised July 12, 2017; accepted November 6, 2017; \\ published March 31, 2018
}

\begin{abstract}
Target-tracking algorithm based on ad hoc camera sensor networks (ACSNs) utilizes the distributed observation capability of nodes to achieve accurate target tracking. A compressed sensing-based multiple-target tracking algorithm (CSMTTA) for ACSNs is proposed in this work based on the study of camera node observation projection model and compressed sensing model. The proposed algorithm includes reconfiguration of observed signals and evaluation of target locations. It reconfigures observed signals by solving the convex optimization of L1-norm least and forecasts node group to evaluate a target location by the motion features of the target. Simulation results show that CSMTTA can recover the subtracted observation information accurately under the condition of sparse sampling to a high target-tracking accuracy and accomplish the distributed tracking task of multiple mobile targets.
\end{abstract}

Keywords: Camera sensor networks, target tracking, compressed sensing, location evaluating

This work is supported in part by the Key Grant Scientific and Technological Planning Project of Guangzhou of China (201704020113), the National Natural Science Foundation of China-Guangdong Province Union Foundation Key Projects (U1401251), and the Grant Scientific and Technological Project of Guangdong Province of China (2017A040405059). 


\section{Introduction}

Traditional wireless sensor networks comprise numerous sensor nodes having communication capability [1]. Meanwhile, ad hoc camera sensor network (ACSN) is a special type of such networks; it consists of camera nodes with strong communication capability; it can observe monitored environment and realize fine-grit and accurate image information gathering and target tracking [2]. ACSN also has broad application prospect in the environment, transportation, military, construction, and many other fields.

The most stringent criterion of target tracking is to track with low delay and high accuracy [3]. In the ACSN for target-tracking application and for improving tracking accuracy, speed, and breadth, camera sensor nodes often utilize mutual communication to realize distributed tracking, including exchanging and fusing of target information of different viewing angles and obtaining target movement tracking information through comprehensive calculations [4]. Some studies have been conducted about target-tracking and node-scheduling algorithms for camera sensor networks. Alberto et al. [5] proposed a cluster-based target-tracking algorithm (CTTA) for camera sensor networks, which includes integrating observed data, selecting cluster members, and alternating cluster heads. Tezcan et al. [6] proposed a detecting algorithm by adjusting the observation direction of camera sensor nodes to optimize coverage quality. This algorithm can decrease the influence of barriers on target detection by adjusting the observation direction of camera nodes. Alaei et al. [7] proposed an energy-efficient collaborative node management algorithm for camera sensor networks; they designed a new node-clustering method based on the vision overlap perception area of nodes. Ercan et al. [8] researched the optimization deployment of multiple camera nodes under the sensor network frame and the issue of node optimal set selection for single target tracking. Jiang et al. [9] extended the directional perception model of rotating camera sensor nodes, defined the problem of minimum rotation angle of moving target with k-level coverage, and proposed a distributed greedy k-level coverage algorithm based on mobile target trajectory prediction.

All the aforementioned target-tracking and node-scheduling algorithms for camera sensor networks do not involve the usage of compressed sensing theory. Compressed sensing is a new sampling theory [10], which is a well-known topic in electronic engineering, especially in signal processing. Compressed sensing technique can reconstruct an original signal by sampling minimal signals [11]. This feature adapts to the information collection mechanism of sensor networks. Network nodes only use simple sensing to acquire few sampling data and then send the data to the sink node. This sink node has unlimited energy and computing capability to reconstruct signals that need considerable calculation. This feature can significantly reduce data sampling quantity and transmission quantity of energy-constrained sensor nodes and thus can decrease network energy consumption. Li et al. [12] proved that compressed sensing can provide new insights into data sampling and acquisition in wireless sensor networks and Internet of Things; they also proposed an efficient clustered sparse reconstruction algorithm for in-network compression to reconstruct data accurately with low energy efficiency. By exploiting the correlation of sensor readings, Yang et al. [13] proposed an energy-efficient distributed data storage scheme for sensor networks based on compressed sensing and network coding.

Few researchers have applied compressed sensing theory to target-tracking application in wireless sensor networks. He et al. [14] changed the problem of multiple-target location based on network grid into the problem of compressed sensing and designed an iterative compressed 
sensing algorithm that can locate multiple targets simultaneously based on the idea of multi-resolution analysis. Zheng et al. [15] proposed a probability sensor management strategy based on compressed sensing nodes, in which each sensor node decides whether it should send the data to the sink node according to a specific probability; the algorithm sets the best probability value that can reconstruct the original data perfectly through a specific strategy. Feng et al. [16] applied compressed sensing to study the target location in wireless sensor networks and changed a multiple-target location into a reconstruction of $\mathrm{M} \times \mathrm{N}$-dimensional vectors with a sparse degree of 1 . However, the data that should be transported by the network cannot be compressed further; thus, the $\mathrm{N} \times \mathrm{M}$ sensing data should be transmitted for the $\mathrm{N}$ sensor nodes and $\mathrm{M}$ targets. The aforementioned algorithms have a large amount of data processing and they do not apply compressed sensing to target tracking for camera sensor networks. The current study proposes a compressed sensing-based multiple-target tracking algorithm (CSMTTA) for ACSN and adopts the method of sparse sampling signal reconstruction and forecasting evaluating node group to improve the accuracy and efficiency of target tracking. The proposed algorithm supports distributed tracking to multiple targets and has low complexity. This algorithm is efficient, can adapt to all types of network deployment, and has the characteristics of low cost and high precision in large-scale camera sensor networks.

The rest of this paper is organized as follows. Section 2 presents the network model, including the node observation projection model and compressed sensing model. Section 3 describes the proposed CSMTTA, which mainly includes two steps, namely, observation information reconstruction and target location evaluation. Section 4 provides the experimental evaluation of the observation information reconstruction, evaluation group selection, and tracking accuracy of CSMTTA using a VC++ network simulation platform. Finally, Section 5 concludes the study.

\section{Network Model}

\subsection{Node Observation Projection Model}

This study adopts the observation projection model of camera sensor node in [17], as shown in Fig. 1.

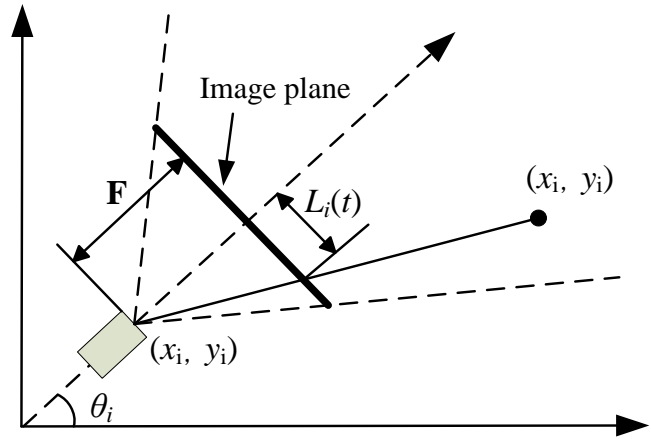

Fig. 1. Projection model of camera node

Suppose that all nodes and targets are in the same plane, the relationship between $L_{i}(t)$ and the observation coordinate by camera node-to-target is as follows: 


$$
L_{i}(t)=\mathbf{F} \cdot \tan \left(\theta_{i}-\arctan \frac{y_{t}-y_{i}}{x_{t}-x_{i}}\right),
$$

where $\mathbf{F}$ is the focal length of the camera sensor node, $\theta_{i}$ is the angle of node lens to $\mathrm{X}$ coordinate axes, and $\left(x_{i}, y_{i}\right)$ is the coordinate of camera sensor node. Target coordinate $\left(x_{t}, y_{t}\right)$ is generated when two camera sensor nodes simultaneously observe a target.

\subsection{Compressed Sensing Model}

If only $k$ non-zero elements exist in a discrete signal, then the signal is considered k-sparse. Suppose that the conversion coefficient of 1D linear original signal $X \in R^{N}$ in certain sparse transform matrix $\Psi \in R^{N \times N}$ is sparse, the conversion coefficient of $\mathrm{X}$ based on $\Psi$ can be described as follows:

$$
\Theta=\Psi^{T} X,
$$

where conversion coefficient $\Theta$ is sparse; thus, most elements in matrix $\Theta$ are 0 .

Subsequently, an $M \times N(M<<N)$-dimensional observation matrix $\Phi$ is designed, which is irrelevant to sparse transform matrix $\Psi$ and can obtain linear observation set $Y$ by observing $\Theta$.

$$
Y=\Phi \Theta=\Phi \Psi^{T} X,
$$

where $Y: M \times 1$ refers to the compressed data. The matrix representation of Formula (3) is shown in Fig. 2.

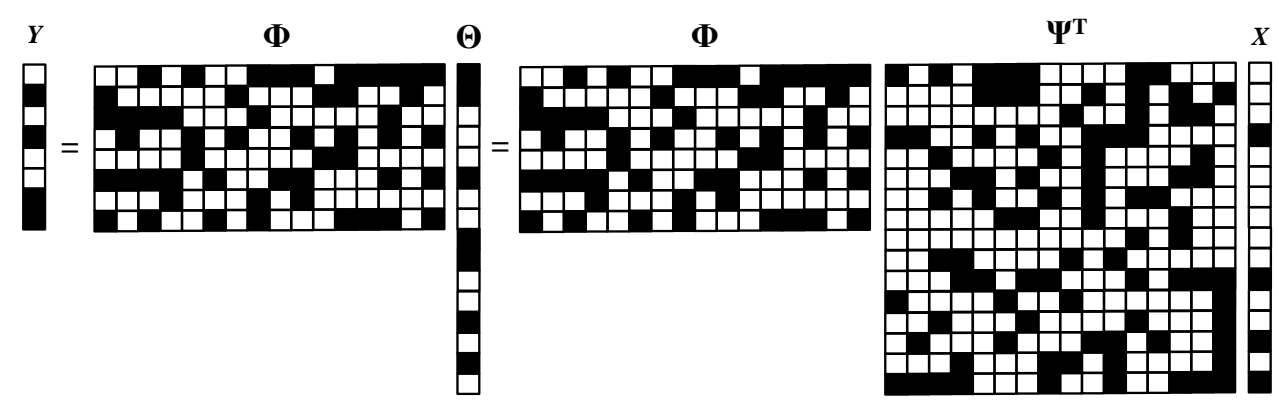

Fig. 2. Matrix representation of compressed sensing

The preceding analysis indicates that the compression of compressed sensing theory uses transform matrix $\Psi$ and metric matrix $\Phi$ to transform $\mathrm{N}$-dimensional signal $\mathrm{X}$ into M-dimensional observation set $\mathrm{Y}$, thereby realizing data compression.

In this study, the element of observation matrix $\Phi \in R^{M \times N}(M<<N)$ is defined as $\varphi_{m, n}$ in ACSN, where $m$ is the $m$ th mobile target in the monitored area $(1 \leq m \leq M)$, and $n$ is the $n$th camera sensor node in the network $(1 \leq n \leq N)$. In the $t$ th round, the observation value of the $n$th node to the $m$ th target is $s_{m, n}(t) \cdot \varphi_{m, n}$ is the difference of $s_{m, n}(t)$ and $s_{m, n}(t-1)$, which is the observation value of the $n$th node to the $m$ th target in the last round. The process of compressed sampling can be expressed as follows: 


$$
\left[\begin{array}{c}
y_{1} \\
y_{2} \\
\vdots \\
y_{M}
\end{array}\right]=\left[\begin{array}{cccc}
\varphi_{1,1} & \varphi_{1,2} & \cdots & \varphi_{1, N} \\
\varphi_{2,1} & \varphi_{2,2} & \cdots & \varphi_{2, N} \\
\vdots & \vdots & \vdots & \vdots \\
\varphi_{M, 1} & \varphi_{M, 2} & \cdots & \varphi_{M, N}
\end{array}\right]\left[\begin{array}{c}
x_{1} \\
x_{2} \\
\vdots \\
x_{N}
\end{array}\right],
$$

where $x_{n}=0$ or 1 when the observation signal of the $n$th node to any target changes in the current round, $x_{n}=1$; otherwise, $x_{n}=0$.

\section{Description of CSMTTA}

\subsection{Observation Information Reconstruction}

In the condition of knowing the observation result of the previous round, CSMTTA aims to recover the location of the moving target in the current round by detecting its observation situation. The algorithm executes subtraction to the observation results in the lower-dimensional compressed observation area.

Under ideal conditions, the observation value of the camera sensor node is the same in the current observation information $s_{n}(t)$ and previous observation information $s_{n}(t-1)$, except for the moving targets in its observation range. Suppose that the total number of node networks in the monitored area is $N$, the observation value of all nodes is placed to the moving targets to connect as a column vector $x$ of size $N$ in sequence and $x(n)(\mathrm{n}=1,2, \ldots, \mathrm{N})$ is used to represent the $n$th element of observing vector $x$. Then, $S(t)=\left\{n|n=l, 2, \ldots, N ;| x_{d}(n) \mid !=0\right\}$ is used to describe the node set with observation values to targets changing in the current round.

In every round, a few sensor nodes can perceive the moving targets. Only few nodes that can reflect the moving target direction and location are required. A wavelet is then selected as the sparse transform matrix, and a difficult threshold process is conducted to the sparse coefficient, thereby leaving the K-largest coefficient and the observation change information that it represents. This process satisfies the precondition of applying compressed sensing theory, which supports the use of subtracted observation information to detect and recover mobile targets in the compressed sensing area.

Suppose that the current-round observation information is $x_{t}$, its compressed observation value is $y_{t}$; and suppose that the previous observation information is $x_{t-1}$, its compressed observation value is $y_{t-1}$. The compressed observation value $y_{d}$ of its subtracted observation information $x_{d}$ can then be represented as follows:

$$
y_{d}=\Phi x_{d}=\Phi\left(x_{t}-x_{t-1}\right)=\Phi x_{t}-\Phi x_{t-1}=y_{t}-y_{t-1} .
$$

The subtracted observation information $x_{d}$ can be recovered by $y_{d}$ via compressed sensing theory; thus, it can also be recovered by $y_{t}$ and $y_{t-1}$.

The preceding compressed sensing model implies that the element in the observation matrix $\Phi \in R^{M \times N}(M<<N)$ is $\varphi_{m, n}$ and that the observation matrix $\Phi$ can be obtained by the actual observation results of each sensor node in the current round. The sum of mobile targets is $M$, 
and the sparse degree of $N$-dimensional vector $x$ is $M$. The observation value variation $y$ of the node is the product of the observation matrix and sparse vector $x$. Therefore, the problem of multiple-target tracking is transformed into a compressed sensing problem of reconstructing $N$-dimensional sparse vector by $M$ measuring results.

Reference [18] assumed that if the observation matrix $\Phi$ satisfies the restricted isometry property (RIP), then $x$ can be reconstructed accurately by solving the optimal $l_{0}$ norm problem, as shown as follows:

$$
\hat{x}=\arg \min \|x\|_{0} \quad \text { s.t. } y=\Phi x,
$$

where $\|\cdot\|_{0}$ represents the $l_{0}$ norm, which is the number of non-zero element in vector $x$. However, solving $l_{0}$ norm minimization is an ill-conditioned problem [19]. Functional analysis and convex optimization theory state that the constraint condition can be lowered and the solution of $l_{0}$ norm can be obtained by optimizing $l_{1}$ norm [20]. Therefore, the optimal point of the objective function in the constrained spaces of $l_{0}$ and $l_{1}$ is almost the same under the condition of sparse signals; thus, $l_{1}$ norm is used for reconstruction and optimization in engineering applications.

$$
\hat{x}=\arg \min \|x\|_{1} \quad \text { s.t. } y=\Phi x
$$

If $x$ is $N$-dimensional vector of sparse degree $S$, then $M$ times to $x$ is randomly measured. The following condition should be satisfied:

$$
M \geq C \mu^{2}(\Phi, \Psi) \cdot S \cdot \log N,
$$

where $C$ is a certain positive constant, and $\mu(\Phi, \Psi)$ represents the coherence of orthogonal matrixes $\Phi$ and $\Psi$ [21].

$$
\mu(\Phi, \Psi)=\sqrt{x} \cdot \max _{1 \leq S, j \leq N}\left|\left\langle\varphi_{k}, \psi_{j}\right\rangle\right|,
$$

and $\mu(\Phi, \Psi)$ satisfies

$$
\mu(\Phi, \Psi) \in[1, \sqrt{N}] .
$$

The observation matrix $\Phi$ then satisfies the RIP.

In CSMTTA,

$$
\mu(\Phi, \Psi) \in \sqrt{N} \cdot \max _{\substack{1 \leq m \leq M \\ 1 \leq n \leq N}} \varphi_{m, n} .
$$

Thus, by solving the convex optimization problem of $l_{1}$ norm minimization, $x$ can be accurately reconstructed using Formula (7).

\subsection{Target Location Evaluation}

CSMTTA supports multiple-target locating and tracking. This algorithm reconstructs the subtracted observation information by the aforementioned compressed sensing method, evaluates node location and motion tendency by camera sensor node observation projection model, and forecasts the node group for evaluating target location. The target location evaluation process is shown in Fig. 3. 


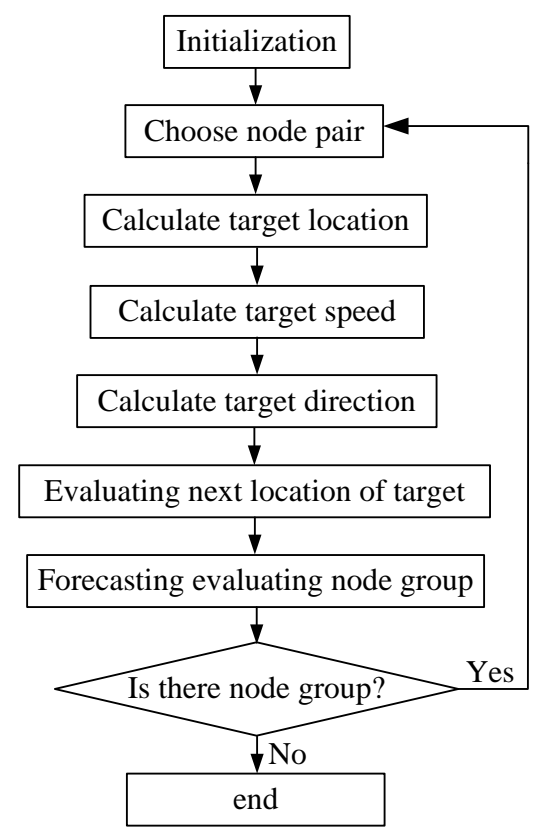

Fig. 3. Target location evaluation flowchart

All camera sensor nodes monitor the location of the target appearance in the monitored area. Once a node monitors the appearance of a target, it immediately broadcasts in the entire network and then starts to perform initialization program and sets up the first evaluation node group of the target location. The node group comprises the nodes that can monitor the target and evaluate the target status using the observation projection model of the camera nodes.

The evaluation node group is divided into several node pairs by calculating from the second group. Each node pair is composed of two camera nodes that can monitor the target. The minimum sum of the hop count of a node pair to the sink node is selected, and the node pair is utilized to calculate the target location, speed, and direction. Location is confirmed by the node observation projection model; speed is confirmed by the distance between the current location and the evaluation location of the previous node pair and the difference in sampling time of these two node pairs; and direction is confirmed by the coordinate relation between the current location and the evaluation location of previous node pair.

The location of the next-round target appearance can be evaluated by location, speed, and direction, and the evaluation node group of the next round can then be forecasted. The evaluation node pair is selected, and the target location is verified according to the actual observation result of the predicted evaluation node group to target, thereby generating the track path of the target.

When many moving targets appear in the monitored area, executing the same program with the above target location evaluation flowchart for each target has no effect for the target tracking among the network nodes.

CSMTTA only executes basic traversal and iterative operations; thus, its computational complexity is low. In the worst condition (e.g., when sufficient targets and observation information of all camera sensor nodes in the current round have variations), its computational complexity is $O(\mathrm{~N})$. 


\section{Performance Evaluation}

VC++ is utilized to design a network simulation platform, in which the target-tracking performance of CSMTTA is evaluated through experiments. Suppose that the camera sensor nodes distribute randomly and densely in a square plane monitored area, the network coverage rate reaches $100 \%$. Multiple targets move randomly in the monitored area, and the sink node lies at the network center, as shown in Fig. 4.

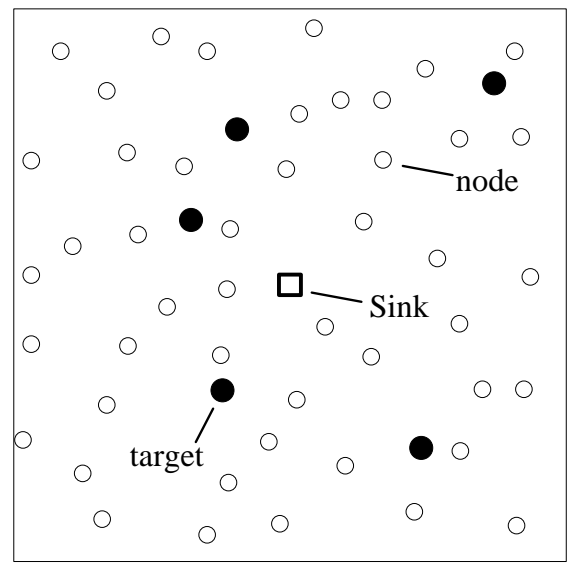

Fig. 4. Network distribution

The image-processing algorithm adopted in this study is an image compression algorithm for wireless camera sensor networks based on FPGA [22]. The default experimental parameters are shown in Table 1.

Table 1. Default experimental parameters

\begin{tabular}{|c|c|}
\hline Parameter & Value \\
\hline Number of nodes & 50 \\
\hline PHY and MAC layers & IEEE $802.15 .4 / 2.4 \mathrm{GHz}$ band \\
\hline $\begin{array}{c}\text { Size of the monitored } \\
\text { area }\end{array}$ & $30 \mathrm{~m} \times 30 \mathrm{~m}$ \\
\hline $\mathbf{F}$ & $8 \mathrm{~mm}$ \\
\hline Visual angle & $120^{\circ}$ \\
\hline Monitoring range & $5 \mathrm{~m}$ \\
\hline Speed of targets & $1 \mathrm{~m} / \mathrm{s}$ \\
\hline
\end{tabular}

\subsection{Observation Information Reconstruction Results}

The recovery of node observation information is initially analyzed using $l_{1}$ norm minimization reconstruction algorithm in the CSMTTA. Target quantity is set to five in the monitored area, and all targets move randomly. Fig. 5 shows the reconstructed results of the observed value of camera sensor nodes in a certain round, which are randomly extracted in the network operation process. The abscissa is the serial number of the node, and the ordinate is the observed node-to-target value in the current round, which is $L_{i}(t)$ in the node-observed projection model. Fig. 5 shows the observed value to targets of 10 in 50 camera sensor nodes. All the 10 nodes can observe targets in the current round. The recovery results of the signal are good, and the average error between the reconstructed and original values of $L_{i}(t)$ is $0.19 \mathrm{~mm}$, which 
indicates that adopting compressed sensing to sample some sparse information can recover the original observed node-to-target value accurately.

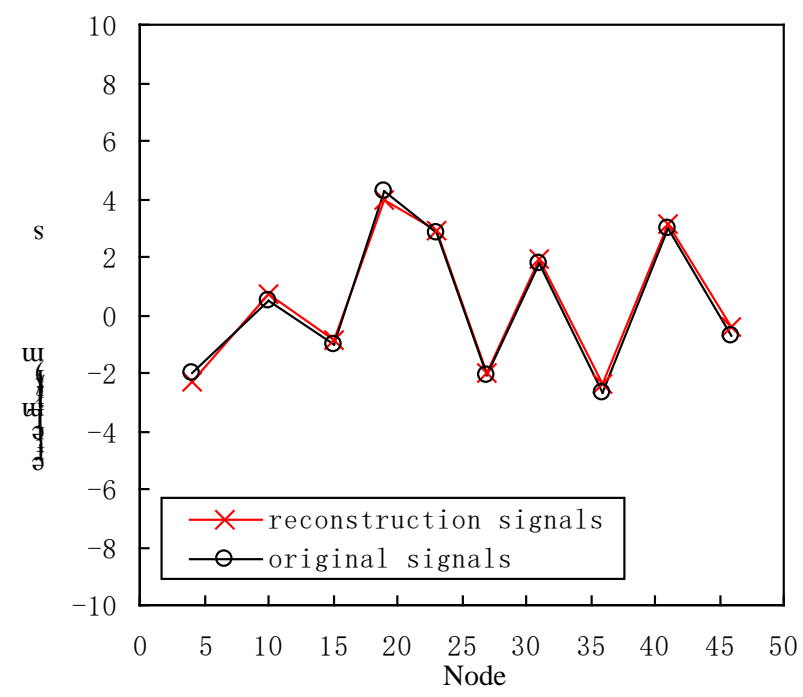

Fig. 5. Reconstructed results of the observed value

\subsection{Evaluation Group}

A simulation experiment is conducted according to the aforementioned experimental parameters, and one round is randomly selected. The observed locations of the five targets and the selection result of the evaluation group of the camera sensor nodes are shown in Fig. 6. The gray sectors are the sensing ranges of the camera sensor nodes.

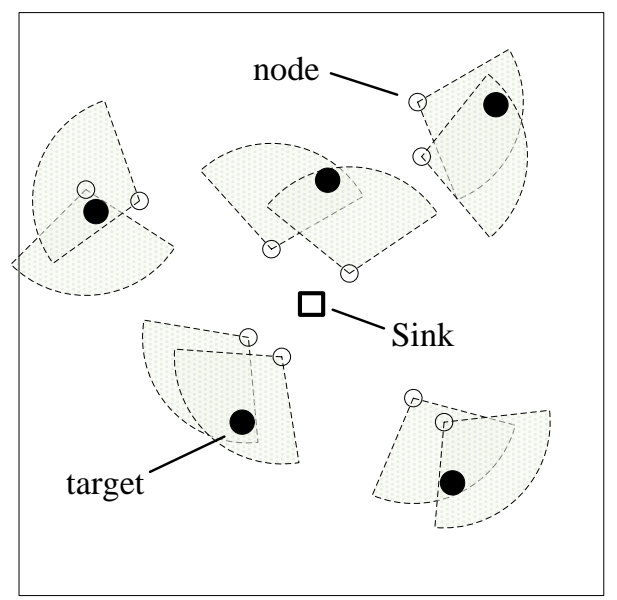

Fig. 6. Node evaluation group

Fig. 6 depicts that the entire situation of the node evaluation group is reasonable. Each target is observed by an evaluation group, including two nodes. The nodes in the evaluation group are generally biased toward the sink node location; thus, the selection of evaluation nodes is affected by the sink node location. The nodes that are close to the sink node easily become evaluation nodes, which have short communication distances to the sink node and can thus effectively reduce network energy consumption. 


\subsection{Tracking Accuracy}

The target number is set as one to evaluate the tracking accuracy of the algorithm to mobile target, and two target moving lines are designed (i.e., straight and curved lines). Their target-tracking situation is shown in Figs. 7(a) and 7(b).

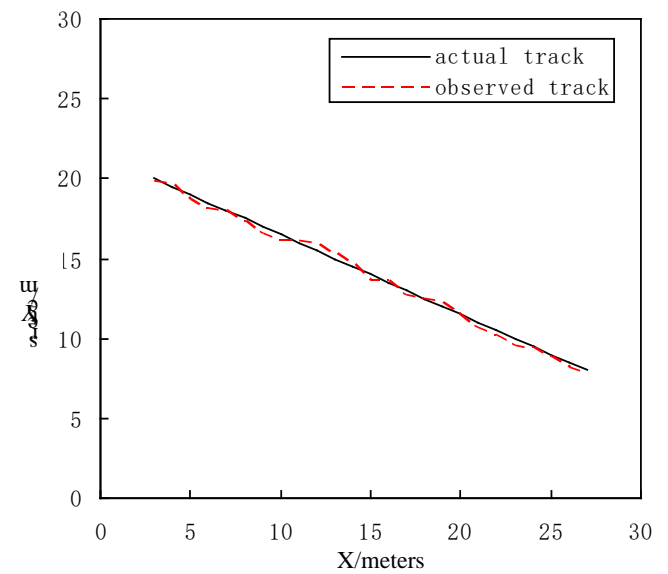

a. Straight line

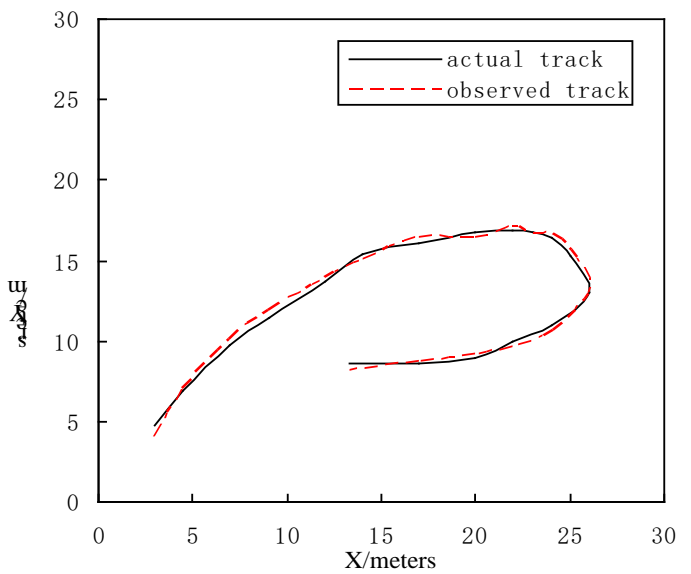

b. Curved line

Fig. 7. Target-tracking trace

Fig. 7 demonstrates the good target-tracking result of the algorithm. Its target-tracking accuracy is higher when the target moving line is straight than when it is curved. CSMTTA evaluates and predicts a straight moving target more accurately. The straight and curved line experiments are repeated five times. A total of 15 fixed sampling points is selected in terms of time, and the mean absolute error of the sampling points is calculated in every experiment. For comparison, the same experiment is conducted to a typical CTTA [5], and its result is shown in Fig. 8. The target-tracking accuracy of CSMTTA is higher by approximately $25 \%$ when the target movement is a straight line than when it is curved, whereas the CTTA is less affected by the curved or straight movement of the target. Overall, the target-tracking accuracy is averagely higher by 35\% in CSMTTA than in the CTTA.

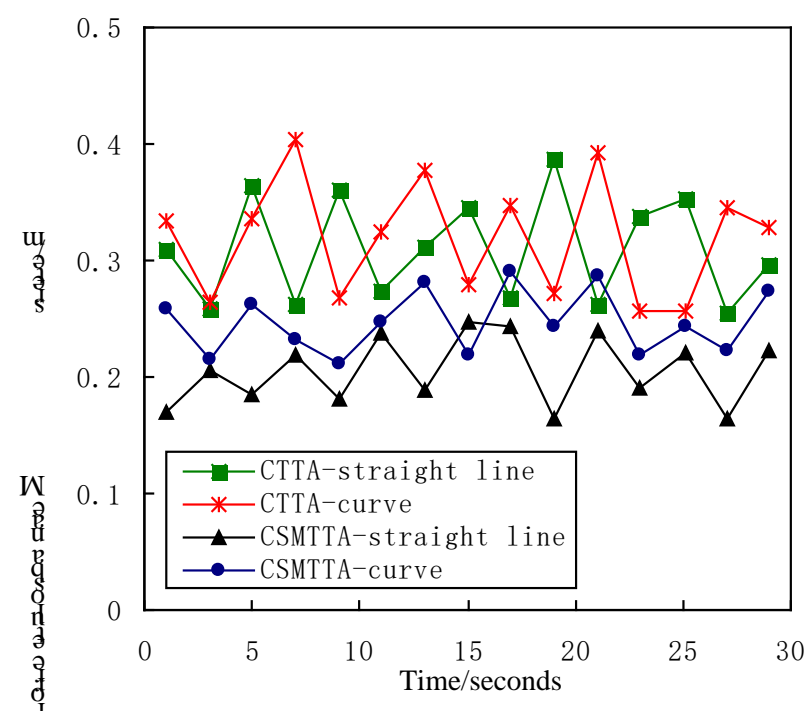

Fig. 8. Target-tracking error 


\subsection{Influence of Target Speed}

In this set of simulation experiments, the behavior of the ACSN is analyzed when the target speed is varied. The target number is set as one, and four different values of target moving speed are selected as follows: $1,2,3$, and $4 \mathrm{~m} / \mathrm{s}$. The other parameters are the initial default values. The influence of target moving speed on tracking accuracy is tested. Similar with the previous experiment, the target movement has two patterns, namely, straight and curved lines. Each experiment is repeated five times, and 15 fixed sampling points are selected in every experiment. The mean absolute error of the sampling points is calculated, and the result is shown in Figs. 9(a) and 9(b).

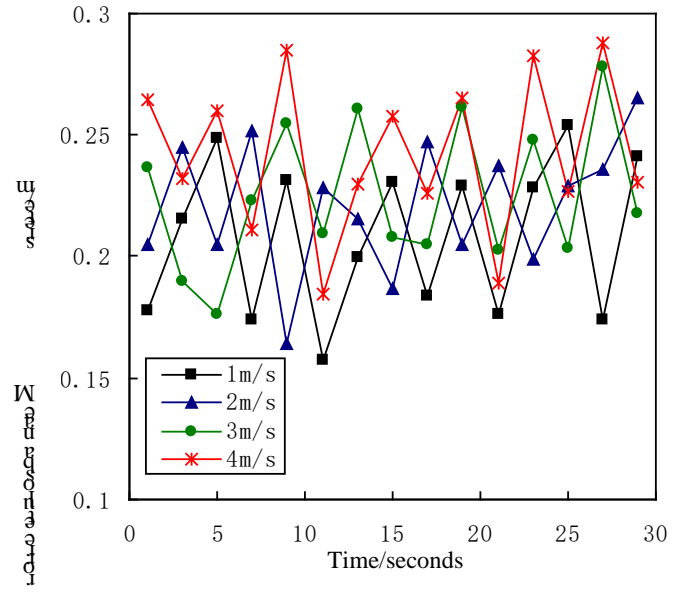

a. Straight line

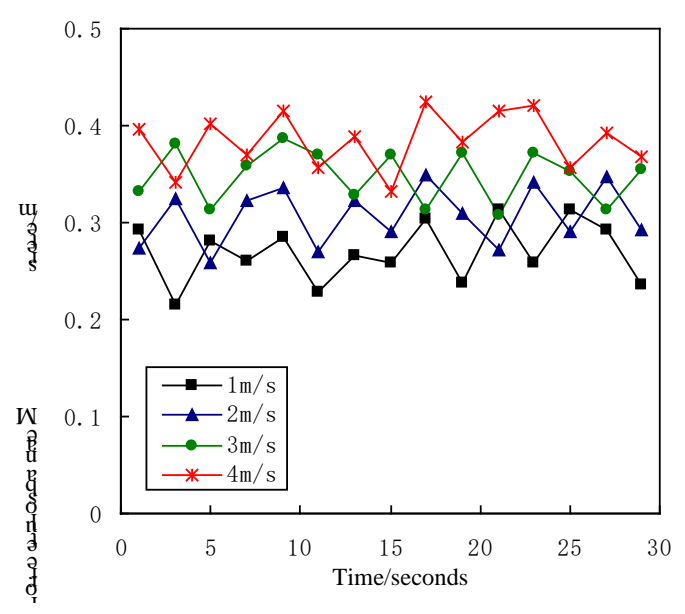

b. Curved line

Fig. 9. Tracking error under different target speeds

When the target movement is a straight line, the influence of target moving speed on tracking accuracy is minimal and can be basically ignored. By contrast, when the target movement is a curved line, the influence of target moving speed on tracking accuracy is relatively significant. Fast target speed results in low tracking accuracy. The tracking error is approximately 1.46 times when the target speed is $4 \mathrm{~m} / \mathrm{s}$ compared with that when it is $1 \mathrm{~m} / \mathrm{s}$. When the target movement is curved, the moving speed is fast; however, the accuracy of CSMTTA forecasting the evaluation group of the camera sensor node is low, thereby leading to decreasing tracking accuracy.

\subsection{Influence of Target Number}

The number of targets is set as 1 to 10 to test the influence of target number variation on algorithm performance and conduct a comparison experiment on target-tracking error. The experimental parameters are the default values. Fig. 10 shows the simulation result of the tracking error. The abscissa is the number of targets, and the ordinate is the mean absolute error. With increasing number of targets, the adopted signal sparseness of the compressed sensing algorithm gradually becomes larger and the mean absolute error gradually increases. Noise of different intensities ( $\mathrm{SNR}=15 \mathrm{~dB}$ and $30 \mathrm{~dB}$ ) are used in the simulation experiment. Fig. 10 shows that the tracking error increases with noise. However, under in the absence of noise, the increasing speed of the tracking error is relatively low with the increasing number of targets. 


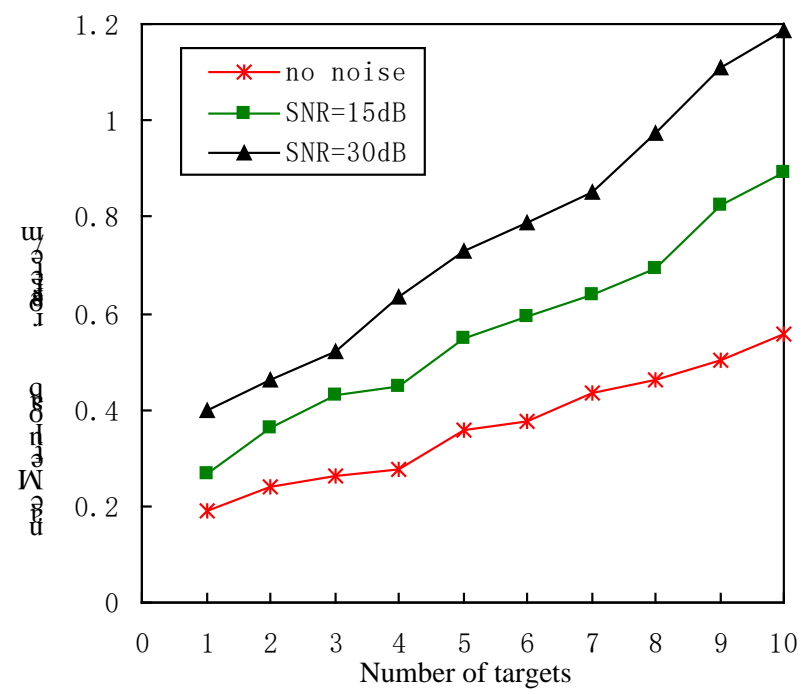

Fig. 10. Influence of target number on tracking error

\section{Conclusion and Future Work}

For the target-tracking algorithm in ACSN, improving target-tracking accuracy and decreasing data sampling quantity are equally important. This study integrates compressed sensing theory and location-forecasting method into a target-tracking algorithm that can effectively reduce data sampling quantity when performing a tracking task. The experimental results show that the proposed CSMTTA can select an appropriate node group when evaluating a target location and recover sparse sampling signal efficiently. Regardless of whether the target moving track is straight or curved, the algorithm has a high target-tracking accuracy, especially under the condition of a straight line. Target moving speed and target quantity affect the algorithm performance. When the target speed or target quantity increases, the tracking accuracy will correspondingly decline. In general, CSMTTA can achieve the multiple-target tracking task efficiently and improve the network performance significantly.

The project team is currently developing mobile nodes with the functions of camera sensing and communication using a wheeled robot as the autonomous mobile device. CSMTTA will be studied and verified under an actual network environment.

\section{References}

[1] C. Cordeiro, D. P. Agrawal, "Wireless sensor networks," Communications of the Acm, vol. 47, no. 6, pp.30-33, 2004. Article (CrossRef Link).

[2] X. Liu. "A survey on wireless camera sensor networks," Frontier and Future Development of Information Technology in Medicine and Education, pp.1085-1094, 2014. Article (CrossRef Link).

[3] V. Tran-Quang, T. Ngo-Quynh and M. Jo, "A lateration-localizing algorithm for energy-efficient target tracking in wireless sensor networks," Ad Hoc \& Sensor Wireless Networks, vol. 34, no.1-4, pp.191-220, 2016. Article (CrossRef Link).

[4] Y. Wang, D. Wang and W Fang, "Automatic node selection and target tracking in wireless camera sensor networks," Computers \& Electrical Engineering, vol. 40, no. 2, pp.484-493, 2014. Article (CrossRef Link). 
[5] A. D. S. Bernabe, "Efficient cluster-based tracking mechanisms for camera-based wireless sensor networks," IEEE Transactions on Mobile Computing, vol. 14, no. 9, pp. 1820-1832, 2015. Article (CrossRef Link).

[6] N. Tezcan, W. Wang, "Self-orienting wireless multimedia sensor networks for occlusion-free viewpoints," Computer Networks, vol. 52, no. 13, pp.2558-2567, 2008. Article (CrossRef Link).

[7] M. Alaei, J. M. Barcelo-Ordinas, "A collaborative node management scheme for energy-efficient monitoring in wireless multimedia sensor networks," Wireless Networks, vol. 19, no. 19, pp.639-659, 2013. Article (CrossRef Link).

[8] A. O. Ercan, D. B. Yang, A. E. Gamal, L. J. Guibas, "Optimal placement and selection of camera network nodes for target localization," Distributed Computing in Sensor Systems, pp.389-404, 2006. Article (CrossRef Link).

[9] W. Shaw, Y. He and I. Lee, "Mobile sink to track multiple targets in wireless visual sensor networks," in Proc. of International Symposium on Ubiquitous Multimedia Computing, pp.51-56, 2008. Article (CrossRef Link).

[10] E. J. Candes. "Compressive sampling," Marta Sanz Sole, vol. 17, no. 2, pp. 1433-1452, 2006. Article (CrossRef Link).

[11] E. J. Candes, M. B. Wakin, "An introduction to compressive sampling," IEEE Signal Processing Magazine, vol. 25, no. 2, pp.21-30, 2008. Article (CrossRef Link).

[12] S. Li, L. D. Xu, X. Wang, "Compressed sensing signal and data acquisition in wireless sensor networks and Internet of Things," IEEE Transactions on Industrial Informatics, vol. 9, no. 4, pp.2177-2186, 2013. Article (CrossRef Link).

[13] X. Yang, X. Tao, E. Dutkiewicz, X. Huang, Y. J. Guo, Q. Cui, "Energy-efficient distributed data storage for wireless sensor networks based on compressed sensing and network coding," IEEE Transactions on Wireless Communications, vol. 12, no.12, pp.5087-5099, 2013. Article (CrossRef Link).

[14] F. H. He, Z. J. Yu, H. T. Liu, "Multiple target localization via compressed sensing in wireless sensor networks," Journal of Electronics \& Information Technology, vol. 34, no. 3, pp.716-721, 2012. Article (CrossRef Link).

[15] Y. J. Zheng, W. Thakshila, K. V. Pramod, "Probabilistic sensor management for target tracking via compressive sensing," in Proc. of IEEE International Conference on Acoustics, Speech and Signal Processing, pp.5075-5079, 2014. Article (CrossRef Link).

[16] C. Feng, S. Valaee, Z. H. Tan, "Multiple target localization using compressive sensing," in Proc. of IEEE Global Communications Conference, pp.1-6, 2009. Article (CrossRef Link).

[17] L. Liu, X. Zhang, H. Ma, "Localization-oriented coverage in wireless camera sensor networks," IEEE Transactions on Wireless Communications, vol. 10, no. 2, pp.484-494, 2011. Article (CrossRef Link).

[18] L. C. Jiao, S. Y. Yang, "Development and prospect of compressive sensing," Journal of Electronic, vol. 39, no. 7, pp.1651-1662, 2011.

[19] D. L. Donnho, Y. Tsaig, "Extensions of compressed sensing," Signal Processing, vol. 86, no. 3, pp.533-548, 2006. Article (CrossRef Link).

[20] E. J. Candes, "The restricted isometry property and its implications for compressed sensing," Comptes Rendus Mathematique, vol. 346, no. 9, pp.589-592, 2008. Article (CrossRef Link).

[21] Candes E J, Y. Plan, "A probabilistic and RIPless theory of compressed sensing," IEEE Transactions on Information Theory, vol. 57, no. 11, pp.7235-7254, 2011. Article (CrossRef Link).

[22] M. L. Kaddachi, L. Makkaoui, A. Soudani, V. Lecuire,, J. M. Moureaux, "FPGA-based image compression for low-power wireless camera sensor networks," in Proc. of International Conference on Next Generation Networks \& Services, pp.68-71, 2011. Article (CrossRef Link). 

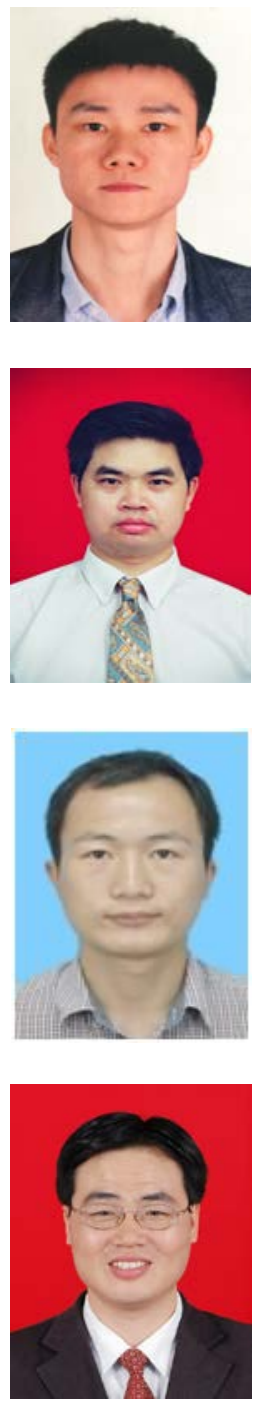

Xu Lu received his M.S. and Ph.D. degrees in Control Theory and Control Engineering from Guangdong University of Technology, China in 2009 and 2015, respectively. He is currently an associate professor in the School of Automation, Guangdong Polytechnic Normal University, China. His research interests include ad hoc camera sensor networks and Internet of things, focusing on target tracking.

Lianglun Cheng received his M.S. degree in Control Theory and Control Engineering from Huazhong University of Science and Technology, China in 1992 and his Ph.D. degree in Control Theory and Control Engineering from the Chinese Academy of Sciences, China in 1999. He is currently a professor and doctoral supervisor in the School of Automation, Guangdong University of Technology, China. He is working in the fields of Internet of things and cyber physical system.

Jun Liu received his M.S. and Ph.D. degrees in Control Theory and Control Engineering from Guangdong University of Technology, China in 2012 and 2015, respectively. He is currently a lecturer in the School of Automation, Guangdong Polytechnic Normal University, China. He is working in the fields of mobile sensor networks and ad hoc network, focusing on sensor deployment.

Rongjun Chen received his M.S. degree in Control Theory and Control Engineering from Guangdong University of Technology, China in 2007 and his Ph.D. degree in Communication and Information System from Sun Yat-sen University, China in 2015. $\mathrm{He}$ is currently an associate professor in the School of Computer Science, Guangdong Polytechnic Normal University, China. His research interests include RFID technology and Internet of things. 